\title{
Bifurcation of a Microelectromechanical Nonlinear Coupling System with Delay Feedback
}

\author{
Yanqiu Li, ${ }^{1}$ Wei Duan, ${ }^{2}$ Shujian $\mathrm{Ma},{ }^{1}$ and Pengfei $\mathrm{Li}^{1}$ \\ ${ }^{1}$ Department of Applied Mathematics, Nanjing University of Technology, Nanjing 211816, China \\ ${ }^{2}$ Petro-China West-East Gas Pipeline Company, Yangzhou 225009, China \\ Correspondence should be addressed to Yanqiu Li; liyanqiul111@gmail.com
}

Received 27 June 2014; Accepted 22 July 2014; Published 18 August 2014

Academic Editor: Junjie Wei

Copyright (c) 2014 Yanqiu Li et al. This is an open access article distributed under the Creative Commons Attribution License, which permits unrestricted use, distribution, and reproduction in any medium, provided the original work is properly cited.

\begin{abstract}
The dynamics of a kind of electromechanical coupling deformable micromirror device torsion micromirror with delay are investigated. Based on the distribution of eigenvalues, we prove that a sequence of Hopf bifurcation occurs at the equilibrium as the delay increases and obtain the critical values of Hopf bifurcation. Explicit algorithms for determining the direction of the Hopf bifurcation and the stability of the bifurcating periodic solutions are derived, using the theories of normal form and center manifold.
\end{abstract}

\section{Introduction}

The microelectromechanical systems (MEMS) represent a very important class of systems having applications in all fields. Because of the nonlinearity and the complexity, modeling and dynamics of MEMS have strongly attracted people's attention [1-4]. MEMS often involve the nonlinear coupling of electrostatic and mechanical physical fields in engineering, so the dynamic characters are complicated. For example, DMD (deformable micromirror device) torsion micromirror, which is widely used in optical communication, optical computing, projection display, and high definition television, is a new type of MEMS based spatial light modulator. The principle is to adjust the distribution of light through controlling the signal, that is, to make the torsional displacement using the electrostatic interaction between the poles so that the spatial light can be modulated. Initially, the scholars usually focus on the static characters of DMD torsion micromirror [5-7]. With the development of microsystem dynamics, there has been some work related to its dynamic characters [8-10].

Our paper is organized as follows. In Section 2, the structure of a kind of electromechanical coupling DMD torsion micromirror and its kinetic equation are given. In Section 3, the previous results about the ordinary differential equation (ODE) are listed. In Section 4, the existence and the critical values of Hopf bifurcation are obtained. In Section 5, the normal form method and the center manifold theory are used to analyze the properties of Hopf bifurcation. In Section 6, we summarize our results.

\section{Description of Model}

The electromechanical coupling structure of DMD torsion micromirror is shown in Figure 1(a). Using angular displacement $\theta$ as generalized coordinate (see Figure 1(b)), the kinetic equation of torsion vibration system can be formed as follows:

$$
I \ddot{\theta}+C_{\theta} \dot{\theta}+K_{\theta} \theta=T,
$$

where $I$ is rotary inertia, $C_{\theta}$ is damping, $K_{\theta}$ is torsional stiffness, $\theta$ is turning angle, and $T$ is the external driving moment of the system.

Based on a series of calculation like $[8,9]$ and setting $\delta=$ $\theta / \theta_{\max }=(a / 2) \theta / d_{0}=a \theta /\left(2 d_{0}\right),(1)$ can be changed into

$$
I \frac{d^{2} \delta}{d t^{2}}+C_{\theta} \frac{d \delta}{d t}+K_{\theta} \delta=\frac{L \varepsilon a^{3} V^{2}}{16 d_{0}^{3} \delta^{2}}\left[\ln (1-\delta)+\frac{\delta}{1-\delta}\right],
$$

where $V$ is the voltage imposed on the electrode, $\varepsilon$ is a dielectric constant of air, $d_{0}$ is the initial distance between two electrodes, and $\theta_{\max }$ is the maximum turning angle as the edge of micromirror plane meets the plate electrode. 


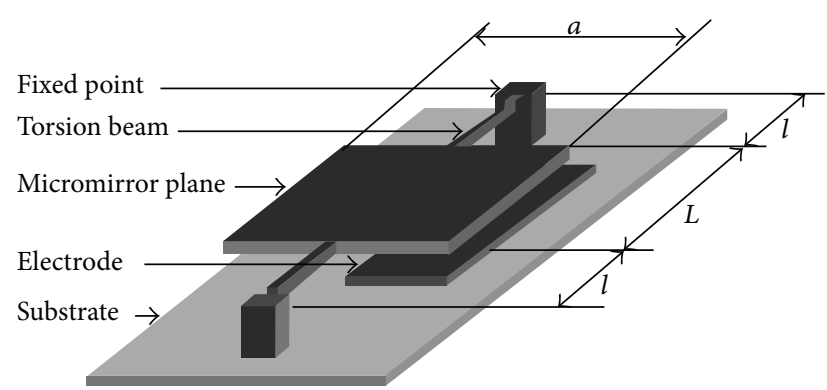

(a)

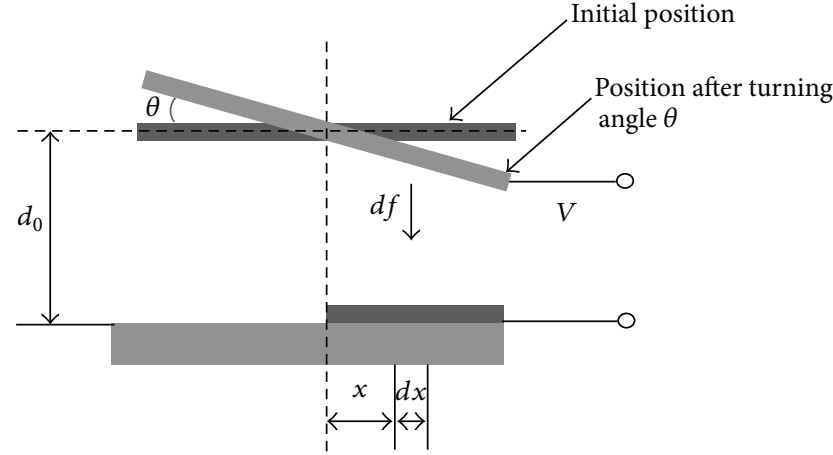

(b)

FIGURE 1: The electromechanical coupling structure of DMD torsion micromirror and the simple scheme for computation.

Introduce the dimensionless time $\tau=\omega_{0} t$ with $\omega_{0}=$ $K_{\theta} / I$. Let $R=C_{\theta} / I \omega_{0}$ and $B=L \varepsilon a^{3} / 16 K_{\theta} d_{0}^{3}$. Then (2) can be rewritten as

$$
\frac{d^{2} \delta}{d t^{2}}+R \frac{d \delta}{d t}+\delta=\frac{B V^{2}}{\delta^{2}}\left[\ln (1-\delta)+\frac{\delta}{1-\delta}\right],
$$

where $\delta=\theta / \theta_{\max }, R$ is a damping coefficient, $V$ is an excitation voltage, and $B$ is a coefficient determined by geometric parameters of the micromirror system. Equation (3) is thus the dimensionless kinetic equation of a simplified physical model of a kind of electromechanical coupling structure of DMD torsion micromirror.

\section{Existence of Equilibria and Bifurcation of ODE}

Now, we transform (3) into the following system:

$$
\begin{aligned}
& \dot{x}=y, \\
& \dot{y}=-R y-x+\frac{B V^{2}}{x^{2}}\left[\ln (1-x)+\frac{x}{1-x}\right],
\end{aligned}
$$

where $x=\delta, y=\dot{x}=d \delta / d t$.

Reference [9] considers the existence and the type of equilibrium of (4) and the appearance of its bifurcation. The conclusions below are gained by [9].

Theorem 1. If $0<B V^{2}<H(\tilde{x})$ holds, then system (4) has two equilibria. If $B V^{2}=H(\tilde{x})$, then there is only one equilibrium in system (4) and no equilibrium exists if $B V^{2}>H(\widetilde{x})$. Here $\tilde{x}$ is the maximum point of $H(x)=x^{3} /(\ln (1-x)+x /(1-x))$ in the interval $(0,1)$.

Theorem 2. Assume that $R \neq 0$ and $X=\left(x^{*}, 0\right)$ is an equilibrium point of system (4). The following results hold.

(1) If $0<x^{*}<\tilde{x}$, then $X=\left(x^{*}, 0\right)$ is a node or focus point of system (4).

(2) If $\tilde{x}<x^{*}<1$, then $X=\left(x^{*}, 0\right)$ is a saddle point of system (4).
(3) If $x^{*}<\tilde{x}$, then $X=\left(x^{*}, 0\right)$ is a saddle-node point of system (4).

Theorem 3. Assume that $X=\left(x^{*}, 0\right)$ is an equilibrium point of system (4). If $0<x^{*}<\tilde{x}$, then a degenerate Hopf bifurcation occurs at the point $X$ as $R=0$.

Theorem 4. Assume that $X=\left(x^{*}, 0\right)$ is an equilibrium point of system (4). If $x^{*}=\tilde{x}$, then Bogdanov-Takens bifurcation occurs at the point $X$ as $R=0$.

\section{Hopf Bifurcation of System with Delay}

Introducing linear time delay feedback for (4), we have

$$
\begin{aligned}
\dot{x}= & y, \\
\dot{y}= & -R y-x+\frac{B V^{2}}{x^{2}}\left[\ln (1-x)+\frac{x}{1-x}\right] \\
& +k(x(t-\sigma)-x(t)),
\end{aligned}
$$

where $\sigma \geq 0$ is the time delay and $k>0$ is the coefficient of feedback gain.

Then $X=\left(x^{*}, 0\right)$ is also an equilibrium point of system (5). Now we begin to consider the stability of the system at the point $X$. The characteristic equation of its corresponding linear system around $X$ is

$$
\lambda(\lambda+R)+M-k e^{-\lambda \sigma}=0,
$$

where $M=3+k-B V^{2} / x^{*}\left(1-x^{*}\right)^{2}$.

Refer to the eigenvalue analysis in [11] and when $\tau=0$,

(6) has roots $\lambda_{1,2}=\left(-R \pm \sqrt{R^{2}-4(M-k)}\right) / 2$.

Lemma 5. (1) If $M>k$, then $\operatorname{Re} \lambda_{1,2}<0$.

(2) If $M=k$, then $\lambda_{1}=0, \lambda_{2}=-R$.

(3) If $M>k$, then $\lambda_{1}>0, \lambda_{2}<0$.

Denote that

$$
\text { (H) } R^{2}<2 M, \quad R^{4}+4\left(k^{2}-M R^{2}\right) \geq 0 .
$$

Lemma 6. Suppose that $M>k$ is satisfied. 
(1) If $(H)$ is not satisfied, then all roots of (6) have negative real parts for any $\sigma \geq 0$.

(2) If $(H)$ is satisfied, then there exists a sequence of $\sigma_{ \pm}^{j}$ satisfying $0<\sigma_{ \pm}^{0}<\sigma_{ \pm}^{1}<\cdots<\sigma_{ \pm}^{n}<\cdots$ such that (6) has a pair of purely imaginary roots $\pm i \omega_{ \pm}$when $\sigma=\sigma_{ \pm}^{j}$, and all roots of (6) have negative real parts when $\sigma \in\left[0, \min \left\{\sigma_{+}^{0}, \sigma_{-}^{0}\right\}\right)$,

where

$$
\begin{gathered}
\omega_{ \pm}^{2}=\frac{-\left(R^{2}-2 M\right) \pm \sqrt{\left(R^{2}-2 M\right)^{2}-4\left(M^{2}-k^{2}\right)}}{2}, \\
\sigma_{ \pm}^{j}=\frac{\arccos \left(\left(\omega_{ \pm}^{2}-M\right) / k\right)+(2 j+1) \pi}{\omega_{ \pm}}, \quad j=0,1,2, \ldots
\end{gathered}
$$

Proof. When $\sigma=0$ and $M>k$, then $\operatorname{Re} \lambda_{1,2}<0$; that is, all roots of (6) have negative real parts.

Let $\pm i \omega(\omega>0)$ be a pair of roots of (6). Substitute $i \omega$ into (6) and separate the real and imaginary parts

$$
\begin{aligned}
& \cos (\omega \sigma)=\frac{M-\omega^{2}}{k}, \\
& \sin (\omega \sigma)=-\frac{R \omega}{k} .
\end{aligned}
$$

Hence,

$$
\begin{aligned}
& \omega_{ \pm}^{2}=\frac{-\left(R^{2}-2 M\right) \pm \sqrt{\left(R^{2}-2 M\right)^{2}-4\left(M^{2}-k^{2}\right)}}{2} . \\
& \text { If }(H) \text { is not satisfied, then } \omega_{ \pm}
\end{aligned}
$$

Hence, (6) does not have pure imaginary roots. The first conclusion is right due to Lemma 2.4 in Wei and Ruan [12]. Define

If $(H)$ is satisfied, then $\omega_{ \pm}$are positive real numbers.

$$
\sigma_{ \pm}^{j}=\frac{\arccos \left(\left(\omega_{ \pm}^{2}-M\right) / k\right)+(2 j+1) \pi}{\omega_{ \pm}}, \quad j=0,1,2, \ldots,
$$

and $\left(\sigma_{ \pm}^{j}, \omega_{ \pm}\right)$satisfies (6). Equation (6) has the roots $\pm i \omega_{ \pm}$ when $\sigma=\sigma_{ \pm}^{j} \cdot \min \left\{\sigma_{+}^{0}, \sigma_{-}^{0}\right\}$ is the first positive value which makes (6) have pure imaginary roots. Hence, all roots of (6) have negative real parts when $\sigma \in\left[0, \min \left\{\sigma_{+}^{0}, \sigma_{-}^{0}\right\}\right)$ due to Lemma 2.4 in Wei and Ruan [12].

The proof is complete.

Let $\lambda(\sigma)=\alpha(\sigma)+i \omega(\sigma)$ be a root of (6) near $\sigma=\sigma_{ \pm}^{j}$ satisfying $\alpha\left(\sigma_{ \pm}^{j}\right)=0, \omega\left(\sigma_{ \pm}^{j}\right)=\omega_{ \pm}, j=0,1,2, \ldots$

Lemma 7. Suppose that $(H)$ is satisfied.
(1) $\operatorname{Re}\left(\left.(d \lambda / d \sigma)\right|_{\lambda=i \omega_{-}, \sigma=\sigma_{-}^{j}}\right)>0$.

(2) $\operatorname{Re}\left(\left.(d \lambda / d \sigma)\right|_{\lambda=i \omega_{+}, \sigma=\sigma_{+}^{j}}\right)=\left\{\begin{array}{l}<0, \text { if } M<1 \text {, } \\ >0, \text { if } M>1 \text {, }\end{array}\right.$

where $j=0,1,2, \ldots$

Proof. Substituting $\lambda(\sigma)$ into (6) and taking the derivative with respect to $\sigma$, we get

$$
\begin{aligned}
\operatorname{Re}\left(\left.\frac{d \lambda}{d \sigma}\right|_{\lambda=i \omega_{ \pm}, \sigma=\sigma_{ \pm}^{j}}\right) \\
=\frac{\omega_{ \pm}^{2}\left(M-\omega_{ \pm}^{2}\right)}{\Delta} \\
=\frac{\omega_{ \pm}^{2}\left(R^{2} \mp \sqrt{\left(R^{2}-2 M\right)^{2}-4\left(M^{2}-k^{2}\right)}\right)}{\Delta},
\end{aligned}
$$

where $\Delta=\left(R+k \sigma_{ \pm}^{j} \cos \omega_{ \pm} \sigma_{ \pm}^{j}\right)^{2}+\left(2 \omega_{ \pm}-k \sigma_{ \pm}^{j} \sin \omega_{ \pm} \sigma_{ \pm}^{j}\right)^{2}, j=$ $0,1,2, \ldots$.

Using the lemmas above, we have Theorem 8.

Theorem 8. Suppose that $M>k$ is satisfied.

(1) If $(H)$ is not satisfied, then $X$ of system (5) is asymptotically stable for any $\sigma \geq 0$.

(2) If $(H)$ is satisfied, then $X$ of system (5) is asymptotically stable when $\sigma \in\left[0, \sigma_{-}^{0}\right)$, where $\sigma_{ \pm}^{j}$ is defined in Lemma 6.

\section{The Direction and Stability of Hopf Bifurcation}

We first rescale the time by $t \mapsto t / \sigma$ to normalize the delay so that system (5) can be written as the form

$$
\begin{aligned}
\dot{x}=\sigma y, & \\
\dot{y}=- & R \sigma y-\sigma\left(x+x^{*}\right)+\frac{\sigma B V^{2}}{\left(x+x^{*}\right)^{2}} \\
\times & {\left[\ln \left(1-\left(x+x^{*}\right)\right)+\frac{x+x^{*}}{1-\left(x+x^{*}\right)}\right] } \\
+ & k \sigma(x(t-1)-x(t)) .
\end{aligned}
$$

Let $\sigma=\sigma_{0}+\mu, \mu \in \mathbb{R}$, and $\sigma_{0} \in\left\{\sigma_{k}(j)\right\}, k=1,2, j=$ $0,1,2, \ldots$. Then $\mu=0$ is the Hopf bifurcation value for (13).

Notating $C=C\left([-1,0], \mathbb{R}^{2}\right)$, by Riesz representation theorem, there exists a matrix whose components are bounded variation functions $\eta(\theta, \mu)(-1 \leq \theta \leq 0)$ such that

$$
L_{\mu}(\varphi)=\int_{-1}^{0} d \eta(\theta, \mu) \varphi(\theta)
$$

where 


$$
\begin{aligned}
A & =\left(\begin{array}{ll}
0 \\
-k-1-\frac{2 B V^{2}}{x^{*^{3}}}\left[\ln \left(1-x^{*}\right)+\frac{x^{*}\left(1-2 x^{*}\right)}{2\left(1-x^{*}\right)^{2}}\right]-R
\end{array}\right), \\
B & =\left(\begin{array}{ll}
0 & 0 \\
k & 0
\end{array}\right), \\
\eta(\theta, \mu) & = \begin{cases}\left(\sigma_{0}+\mu\right) A, & \theta=0, \\
0, & \theta \in(-1,0), \\
-\left(\sigma_{0}+\mu\right) B, & \theta=-1 .\end{cases}
\end{aligned}
$$

Denote

$$
\begin{aligned}
F(\mu, \varphi)= & \left(\sigma_{0}+\mu\right)\left(\begin{array}{c}
0 \\
k_{1}
\end{array}\right) \varphi^{2}(0) \\
& +\left(\sigma_{0}+\mu\right)\left(\begin{array}{c}
0 \\
k_{2}
\end{array}\right) \varphi^{3}(0)+\cdots,
\end{aligned}
$$

where

$$
\begin{aligned}
& k_{1}=\frac{3 B V^{2}}{x^{*^{4}}}\left[\ln \left(1-x^{*}\right)+\frac{x^{*}\left(2-5 x^{*}+4 x^{*^{2}}\right)}{3\left(1-x^{*}\right)^{3}}\right], \\
& k_{2}=-\frac{4 B V^{2}}{x^{*^{5}}}\left[\ln \left(1-x^{*}\right)+\frac{x^{*}\left(11-28 x^{*}+13 x^{*^{2}}\right)}{12\left(1-x^{*}\right)^{3}}\right] .
\end{aligned}
$$

For $\varphi \in C^{1}\left([-1,0], \mathbb{R}^{2}\right)$, define

$$
\begin{gathered}
A(\mu) \varphi= \begin{cases}\frac{d \varphi(\theta)}{d \theta}, & \theta \in[-1,0), \\
\int_{-1}^{0} d \eta(t, \mu) \varphi(t), & \theta=0,\end{cases} \\
R(\mu) \varphi= \begin{cases}0, & \theta \in[-1,0), \\
F(\mu, \varphi), & \theta=0 .\end{cases}
\end{gathered}
$$

Equation (13) can be written as

$$
\dot{u}_{t}=A(\mu) u_{t}+R(\mu) u_{t}
$$

where $u=(x, y)^{T}$ and $u_{t}=u(t+\theta), \theta \in[-1,0]$.

For $\psi \in C^{1}\left([0,1], \mathbb{R}^{2}\right)$, define

$$
A^{*} \psi(s)= \begin{cases}\frac{-d \psi(s)}{d s}, & s \in(0,1], \\ \int_{-1}^{0} d \eta(t, 0) \psi(-t), & s=0 .\end{cases}
$$

For $\varphi \in C^{1}\left([-1,0], \mathbb{R}^{2}\right)$ and $\psi \in C^{1}\left([0,1], \mathbb{R}^{2}\right)$, define the bilinear form

$$
\langle\psi, \varphi\rangle=\bar{\psi}(0) \varphi(0)-\int_{-1}^{0} \int_{\xi=0}^{\theta} \bar{\psi}(\xi-\theta) d \eta(\theta) \varphi(\xi) d \xi,
$$

where $\eta(\theta)=\eta(\theta, 0)$. Then $A^{*}$ and $A(0)$ are adjoint operators, and $\pm i \sigma_{0} \omega_{0}$ are eigenvalues of $A(0)$. Thus, they are also eigenvalues of $A^{*}$.

Let $q(\theta), q^{*}(s)$ satisfying $\left\langle q^{*}, q\right\rangle=1$ and $\left\langle q^{*}, \bar{q}\right\rangle=0$ be eigenvectors of $A, A^{*}$ corresponding to $i \sigma_{0} \omega_{0}$ and $-i \sigma_{0} \omega_{0}$, respectively. By direct computation, we obtain that

$$
\begin{aligned}
q(\theta) & =\left(1, i \omega_{0}\right)^{T} e^{i \sigma_{0} \omega_{0} \theta} \\
q^{*}(s) & =\frac{1}{k e^{i \sigma_{0} \omega_{0}}-R}\left(i \omega_{0}-R, 1\right) e^{i \sigma_{0} \omega_{0} s} .
\end{aligned}
$$

Using the same notation as in Hassard et al. [13], define

$$
z(t)=\left\langle q^{*}, u_{t}\right\rangle, \quad w(t, \theta)=u_{t}(\theta)-2 \operatorname{Re}\{z(t) q(\theta)\} .
$$

On the center manifold $\mathscr{C}_{0}$, we have

$$
w(t, \theta)=w(z(t), \bar{z}(t), \theta)
$$

where

$$
w(z(t), \bar{z}(t), \theta)=w_{20}(\theta) \frac{z^{2}}{2}+w_{11}(\theta) z \bar{z}+w_{02} \frac{\bar{z}^{2}}{2}+\cdots
$$

$z$ and $\bar{z}$ are local coordinates for the center manifold $\mathscr{C}_{0}$ in the direction of $q^{*}$ and $\bar{q}^{*}$. Notice that $w$ is real if $u_{t}$ is real. We only consider real solutions. Since $\mu=0$, we have

$$
\begin{aligned}
\dot{z}( & t) \\
& =i \tau_{0} \omega_{0} z+\left\langle q^{*}(s), f(0, w+2 \operatorname{Re}\{z(t) q(\theta)\})\right\rangle \\
& =i \tau_{0} \omega_{0} z+\bar{q}^{*}(0) f(0, w(z, \bar{z}, 0)+2 \operatorname{Re}\{z(t) q(0)\}) \\
& \stackrel{\text { def }}{=} i \tau_{0} \omega_{0} z(t)+\bar{q}^{*}(0) f_{0}(z, \bar{z})
\end{aligned}
$$

for solution $u_{t} \in \mathscr{C}_{0}$. We rewrite this as

$$
\dot{z}(t)=i \tau_{0} \omega_{0} z(t)+g(z, \bar{z}),
$$


where

$$
\begin{gathered}
f_{0}(z, \bar{z})=f_{z^{2}} \frac{z^{2}}{2}+f_{\bar{z}^{2}} \frac{\bar{z}^{2}}{2}+f_{z \bar{z}} z \bar{z}+f_{z^{2} \bar{z}} \frac{z^{2} \bar{z}}{2}+\cdots, \\
g(z, \bar{z}) \\
=\bar{q}^{*}(0) f(0, w(z, \bar{z}, 0)+2 \operatorname{Re}\{z(t) q(0)\}) \\
=g_{20} \frac{z^{2}}{2}+g_{11} z \bar{z}+g_{02} \frac{\bar{z}^{2}}{2}+g_{21} \frac{z^{2} \bar{z}}{2}+\cdots .
\end{gathered}
$$

Comparing the coefficients of (26) and (27) and noticing (29), we have

$$
\begin{aligned}
& g_{20}=\bar{q}^{*}(0) f_{z^{2}}, \\
& g_{11}=\bar{q}^{*}(0) f_{z \bar{z}}, \\
& g_{02}=\bar{q}^{*}(0) f_{\bar{z}^{2}}, \\
& g_{21}=\bar{q}^{*}(0) f_{z^{2} \bar{z}} .
\end{aligned}
$$

By

$$
\begin{aligned}
w(z, \bar{z}, \theta) & =u_{t}(\theta)-2 \operatorname{Re}(z(t) q(\theta)) \\
& =u_{t}-z(t) q(\theta)-\bar{z}(t) \bar{q}(\theta),
\end{aligned}
$$

then

$$
\begin{aligned}
u_{t}(\theta)= & \left(1, i \omega_{0}\right)^{T} e^{i \sigma_{0} \omega_{0} \theta} z(t)+\left(1,-i \omega_{0}\right)^{T} e^{-i \sigma_{0} \omega_{0} \theta} \bar{z}(t) \\
& +w_{20}(\theta) \frac{z^{2}}{2}+w_{11}(\theta) z \bar{z}+w_{02}(\theta) \frac{\bar{z}^{2}}{2}+\cdots,
\end{aligned}
$$

where $w_{i j}(\theta)=\left(w_{i j}^{1}(\theta), w_{i j}^{2}(\theta)\right)^{T}$.

By

$$
f_{0}=\sigma_{0}\left(\begin{array}{c}
0 \\
k_{1}
\end{array}\right) \varphi^{2}(0)+\sigma_{0}\left(\begin{array}{c}
0 \\
k_{2}
\end{array}\right) \varphi^{3}(0)+\cdots,
$$

we have

$$
\begin{aligned}
& g_{20}=g_{11}=g_{02}=2 k_{1}, \\
& g_{21}=2 k_{1}\left(w_{20}^{1}(0)+2 w_{11}^{1}(0)\right)+6 k_{2},
\end{aligned}
$$

where

$$
\begin{aligned}
w_{20}^{1}(0)=\frac{8 k_{1} i}{3 \sigma_{0} \omega_{0}} & \\
+2 k_{1} \times\left(k+1+\frac{2 B V^{2}}{x^{*^{3}}}\right. & \\
& \times\left[\ln \left(1-x^{*}\right)+\frac{x^{*}\left(1-2 x^{*}\right)}{2\left(1-x^{*}\right)^{2}}\right] \\
& \left.-k e^{-2 i \sigma_{0} \omega_{0}}+2 i \omega_{0}\left(R+2 i \omega_{0}\right)\right)^{-1},
\end{aligned}
$$

$$
\begin{aligned}
w_{11}^{1}(0)=2 k_{1} \times(1 & +\frac{2 B V^{2}}{x^{*^{3}}} \\
& \left.\times\left[\ln \left(1-x^{*}\right)+\frac{x^{*}\left(1-2 x^{*}\right)}{2\left(1-x^{*}\right)^{2}}\right]\right)^{-1} .
\end{aligned}
$$

Define

$$
\begin{aligned}
C_{1}(0) & =\frac{i}{2 \omega_{0} \sigma_{0}}\left(g_{11} g_{20}-2\left|g_{11}\right|^{2}-\frac{\left|g_{02}\right|^{2}}{3}\right)+\frac{g_{21}}{2}, \\
\operatorname{Re} C_{1}(0) & =\frac{\operatorname{Re} g_{21}}{2} \\
\mu_{2} & =-\frac{\operatorname{Re} C_{1}(0)}{\operatorname{Re}\left(\lambda^{\prime}\left(\sigma_{0}\right)\right)}, \\
\beta_{2} & =2 \operatorname{Re}_{1}(0) .
\end{aligned}
$$

We have $\operatorname{Re}\left(\lambda^{\prime}\left(\sigma_{0}\right)\right) \neq 0$ from Lemma 6 , so the following conclusion is right.

Theorem 9. Suppose that $(H)$ is satisfied.

(1) If $\operatorname{Re}\left(\lambda^{\prime}\left(\sigma_{0}\right)\right)<0$ and $\beta_{2}<0\left(\beta_{2}>0\right)$, then the bifurcating periodic solution is orbitally asymptotically stable (unstable) and the direction of Hopf bifurcation is $\sigma<\sigma_{0}\left(\sigma>\sigma_{0}\right)$.

(2) If $\operatorname{Re}\left(\lambda^{\prime}\left(\sigma_{0}\right)\right)>0$ and $\beta_{2}<0\left(\beta_{2}>0\right)$, then the bifurcating periodic solution is orbitally asymptotically stable (unstable) and the direction of Hopf bifurcation is $\sigma<\sigma_{0}\left(\sigma>\sigma_{0}\right)$.

\section{Conclusion}

In this paper, we investigate the Hopf bifurcation of a kind of electromechanical coupling DMD torsion micromirror with delay feedback. Using the normal form method for functional differential equations (FDEs) and the center manifold theory in [13], we have obtained the properties of Hopf bifurcation.

Because the microscale exists, the pull-in phenomena may be caused between microdevices based on the static electricity effect [14]. Electrostatic pull-in is a very important character of MEMS dynamics, and it has significant impact on electrostatic driven microstructure design. For example, the pull-in phenomena should be avoided to prevent leakage of driving mode of energy while designing the microcomb drive $[15,16]$; we need to use the pull-in character to control the structure of switch for the design of the microswitch [17]. Pull-in instability is a common problem for microstructural vibration, and it limits the operating range (deformation and voltage) to reduce the safety and reliability of microsystem. So we hope to avoid the phenomenon whether to utilize it or not in the design. From the dynamic perspective, the pull-in between microdevices is corresponding to generalized instability of nonlinear system; that is, the amplitude of vibration system exceeds a certain threshold, and the escape 
phenomenon occurs [18]. Our results indicate that the time delay feedback can make the equilibrium or the periodic solution be stable; thus the pull-in phenomenon is restrained well.

\section{Conflict of Interests}

The authors declare that there is no conflict of interests regarding the publishing of this paper.

\section{Acknowledgment}

Projects 11301263 and 41101509 are supported by NSFC.

\section{References}

[1] H. S. Haghighi and A. H. D. Markazi, "Chaos prediction and control in MEMS resonators," Communications in Nonlinear Science and Numerical Simulation, vol. 15, no. 10, pp. 3091-3099, 2010.

[2] M. S. Siewe and U. H. Hegazy, "Homoclinic bifurcation and chaos control in MEMS resonators," Applied Mathematical Modelling, vol. 35, no. 12, pp. 5533-5552, 2011.

[3] T. Bechtold, G. Schrag, and L. H. Feng, System-Level Modeling of MEMS, Wiley-VCH, Weinheim, Germany, 2013.

[4] Y. Yu and Z. Mi, "Dynamic modeling and control of electromechanical coupling for mechanical elastic energy storage system," Journal of Applied Mathematics, vol. 2013, Article ID 603063, 11 pages, 2013.

[5] O. Degani, E. Socher, A. Lipson et al., "Pull-in study of an electrostatic torsion microactuator," Journal of Microelectromechanical Systems, vol. 7, no. 4, pp. 373-379, 1998.

[6] O. Degani and Y. Nemirovsky, "Design considerations of rectangular electrostatic torsion actuators based on new analytical pull-in expressions," Journal of Microelectromechanical Systems, vol. 11, no. 1, pp. 20-26, 2002.

[7] X. M. Zhang, F. S. Chau, C. Quan, Y. L. Lam, and A. Q. Liu, "A study of the static characteristics of a torsional micromirror," Sensors and Actuators, A: Physical, vol. 90, no. 1-2, pp. 73-81, 2001.

[8] L. L. Chen, J. P. Zhao, and H. L. Chen, "Stability of equilibrium points of a micro-electromechanical nonlinear coupling system," Chinese Journal of Applied Mechanics, vol. 20, no. 4, pp. 70-74, 2003.

[9] Q. H. Li, J. Z. Xi, and C. C. Hua, "Bifurcations of a microelectromechanical nonlinear coupling system," Communications in Nonlinear Science and Numerical Simulation, vol. 16, no. 2, pp. 769-775, 2011.

[10] N. Lobontiu, Dynamics of Microelectromechanical Systems, Springer, New York, NY, USA, 2007.

[11] S. Ruan and J. Wei, "On the zeros of transcendental functions with applications to stability of delay differential equations with two delays," Dynamics of Continuous, Discrete \& Impulsive Systems A, vol. 10, no. 6, pp. 863-874, 2003.

[12] J. J. Wei and S. G. Ruan, "Stability and bifurcation in a neural network model with two delays," Physica D: Nonlinear Phenomena, vol. 130, no. 3-4, pp. 255-272, 1999.

[13] B. Hassard, N. Kazarinoff, and Y. Wan, Theory and Application of Hopf Bifurcation, Cambridge University Press, 1981.
[14] J. Jia, H. Wang, and K. Fan, "Critical voltage of thin beam driven by electrostatic force," Chinese Journal of Applied Mechanics, vol. 22, no. 1, pp. 95-98, 2005.

[15] B. L. Wang, S. J. Zhou, J. F. Zhao, and X. Chen, "Size-dependent pull-in voltage of electrostatically actuated MEMS," Chinese Journal of Solid Mechanics, vol. 32, no. 6, pp. 541-548, 2011.

[16] W. M. Zhang, M. Guang, and J. B. Zhou, "Nonlinear dynamic characteristics of electrostatically actuated MEMS resonant sensors under parametric excitation," Chinese Quarterly of Mechanics, vol. 30, no. 1, pp. 44-48, 2009.

[17] F. Ding and T. Cao, "Static model for analyzing a micromachined switch consisting of a cantilever beam and its application," Journal of Mechanical Strength, vol. 27, no. 4, pp. 460-464, 2005.

[18] H. L. Shang and Y. P. Wen, "Pull-in stability of electrostatically actuated MEMS resonant sensor and its control," Journal of Vibration, vol. 32, no. 15, pp. 8-13, 2013. 


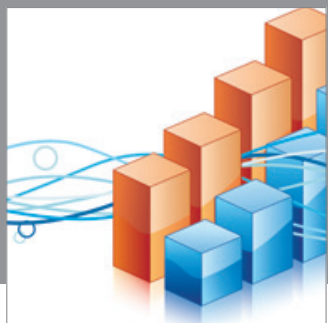

Advances in

Operations Research

mansans

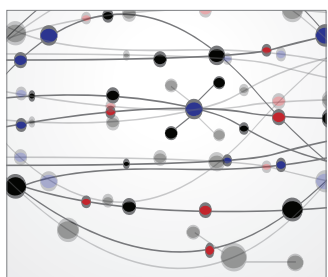

The Scientific World Journal
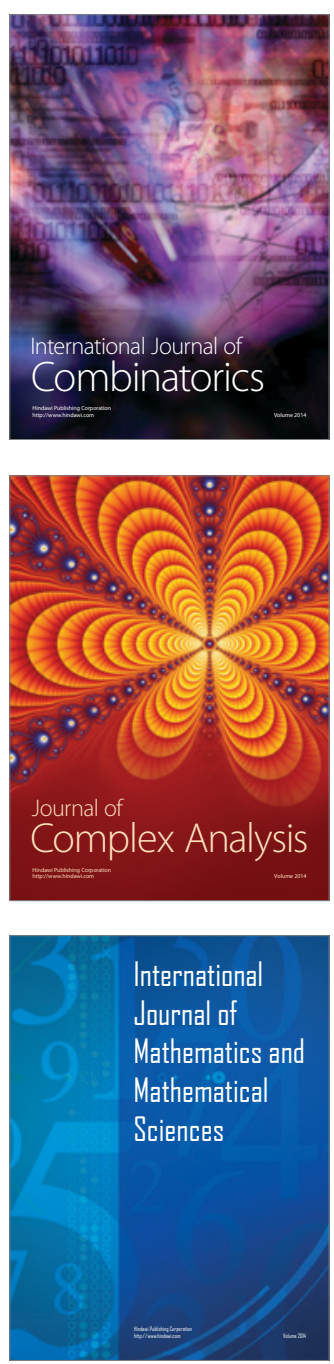
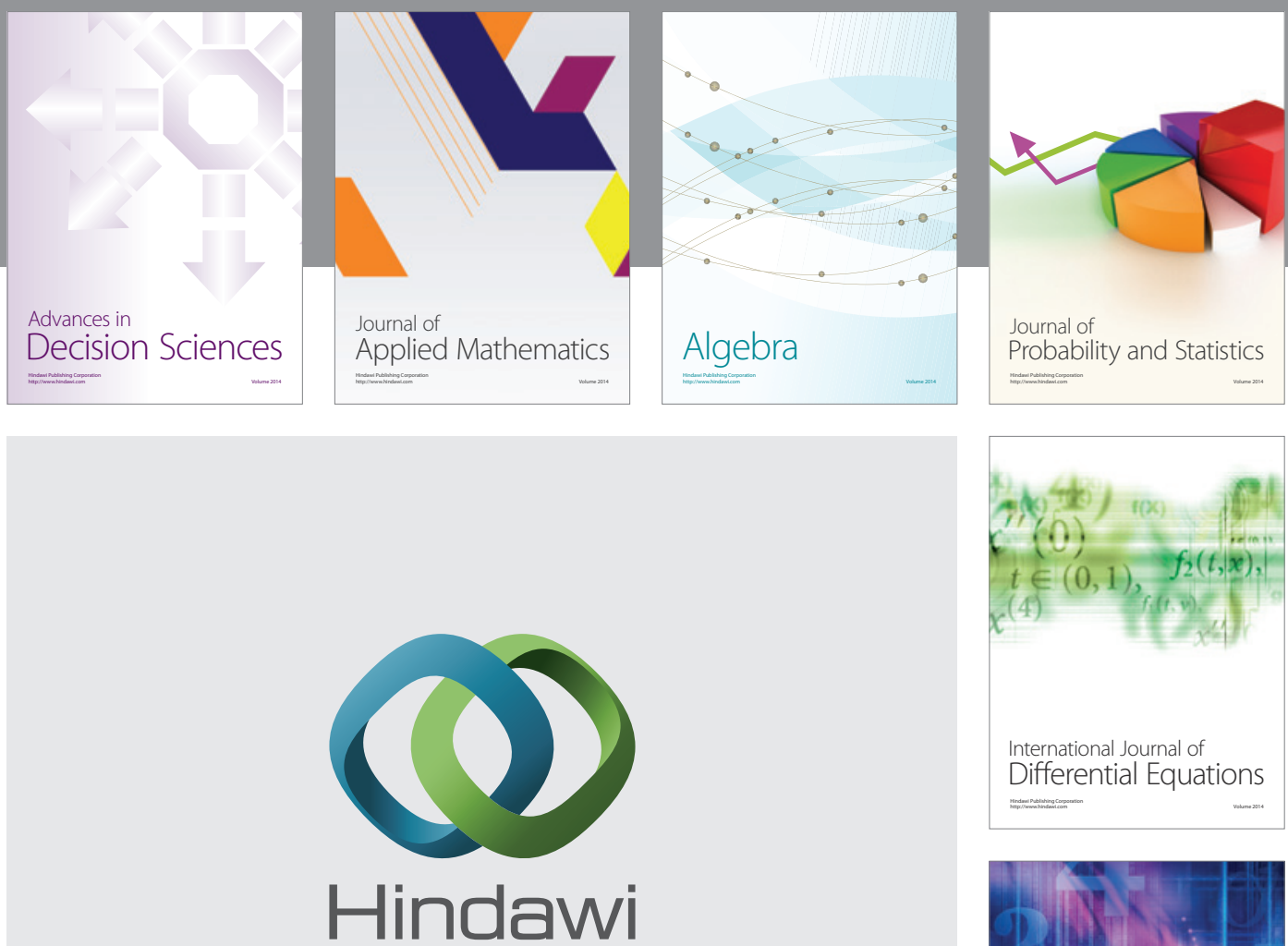

Submit your manuscripts at http://www.hindawi.com
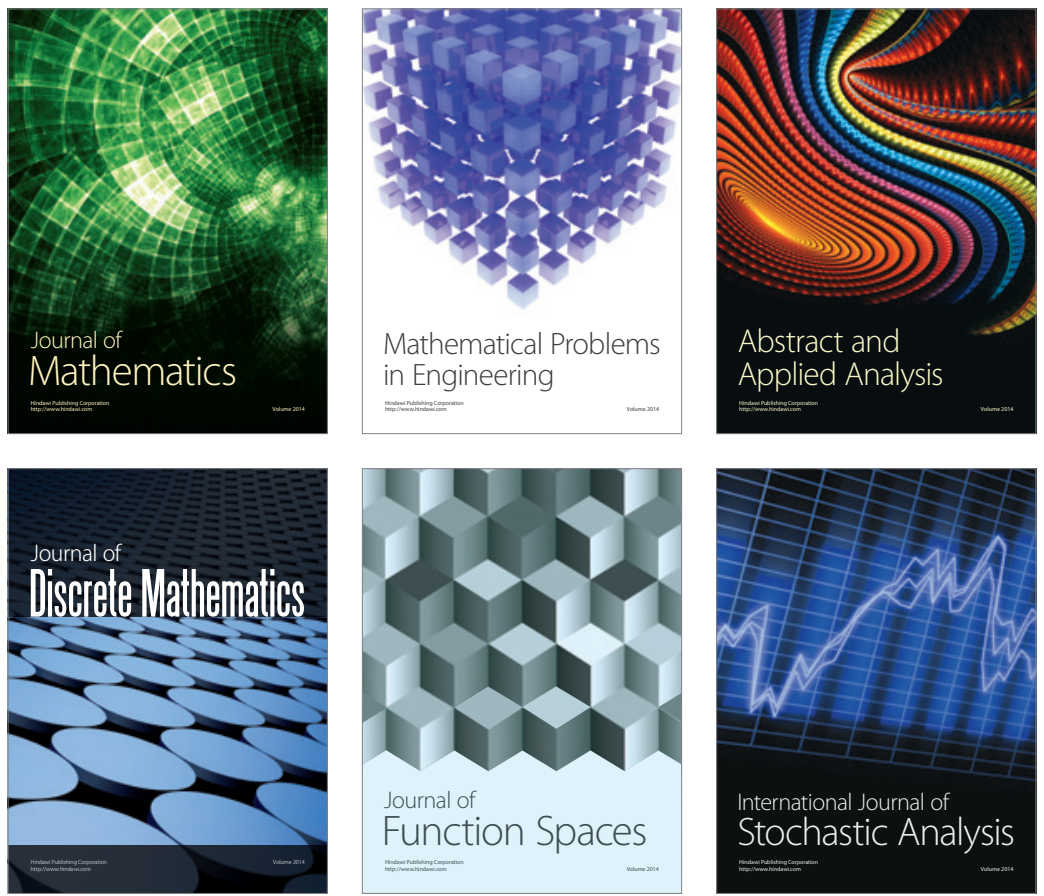

Journal of

Function Spaces

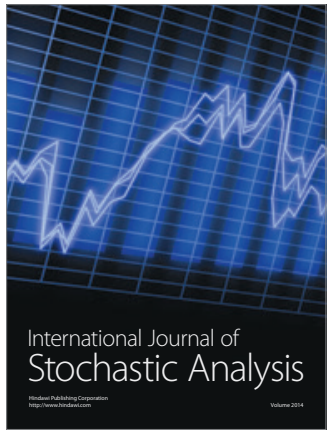

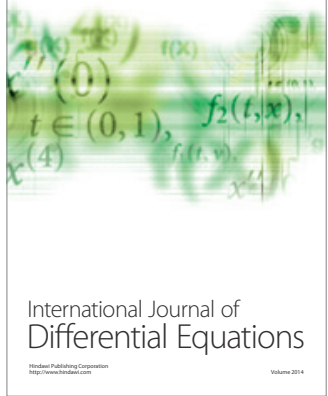
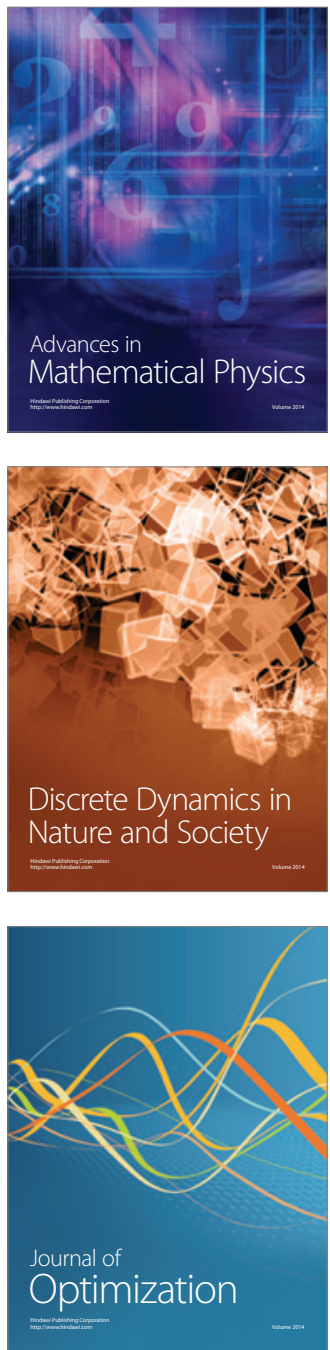